\title{
Über Schallschädigungen nach Erkrankungen des Mittelohres.
}

\author{
Von \\ Oberstabsarzt Dr. Rhese, \\ Privatdozent an der Universität Königsberg i. Pr.
}

Die alte Auffassung, daß ein erkranktes oder erkrankt gewesenes Ohr durch Schallschädigungen in stärkerer Weise beeinträchtigt wird wie ein gesundes, ist neuerdings in Frage gestellt worden. Auf Grund klinischer Beobachtung ist Reinking zu der Ansicht gelangt, daß eine Mittelohrerkrankung einen gewissen Schutz gegen Schallschädigungen gewährt, und $\checkmark$. Eicken hat Tiere durch einseitige Amboßextraktion auf dem entamboßten Ohr vor der degenerativen Neuritis des peripheren Neurons bewahrt. Es handelt sich hierbei nicht nur um die Frage, welche Rolle Luft- und Knochenleitung bei der Entstehung von Schallschädigungen spielen, sondern auch um einen Gegenstand von ungemein praktischer Wichtigkeit für jeden Begutachter Ohrenkranker. Es schien mir deshalb die Feststellung von Wichtigkeit, welche Rolle geringe Schädigungen des Mittelohres in dieser Hinsicht spielen. Um in diese Frage Einsicht zu gewinnen, untersuchte ich zwei Gruppen von Fußartilleristen.

I. 15 Leute, die mit völlig normalem Trommelfell sowie mit normaler Hörfähigkeit für Flüstersprache eingestellt wurden und niemals ohrenkrank waren.

II. $15 \mathrm{Fu}$ Bartilleristen, die bei der Einstellung mit Residuen von Mittelohraffektionen behaftet waren (Narben, größere Atrophieen, Verkallkungen, Perforationen, starke Einziehungen). Die Fälle sowohl von Gruppe I wie von Gruppe II standen sämt. lich am Schluß des zweiten Dienstjahres.

Die Untersuchung betraf Flüstersprache, obere und untere Tongrenze, Schwabach mit kl. c vom Scheitel, Hördauer für $c_{4}$ und $c_{5}$ in Luftleitung. 
Ergebnisse. 1. Eine längere Dienstzeit bei der Fußartillerie hinterläßt fast stets gewisse Einwirkungen auf die Funktion des Kochlearis. Diese Feststellung steht im Einklang mit den bekanntenUntersuchungsergebnissen vonFried rich undJa e hne. Es finden sich daher durch weg so wohl bei den Fällen der Gruppe I wie bei denen der. Gruppe II gewisse Schädigungen der Hördauer von $c_{4}$ oder $c_{5}$ oder beider; es befindet sich sowohl boi Gruppe I wie bei Gruppe II nur je ein Fall, der sowohl $c_{4}$ wie $c_{5}$ bis zum Verklingen hört. Erheblich sind aber hinsichtlich des Grades der gesetzten Schädigungen zwischen beiden Gruppen die Untersehiede.

2. Die Hördauer für $c_{4}$ zeigte eine starke Verkürzung (d. h. unter $60 \%$ ) bei Gruppe I $4 \mathrm{mal}$, bei Gruppe II $7 \mathrm{mal}$.

Die Hördauer für $c_{5}$ zeigte eine starke Verkürzung (d. h. $60 \%$ und weniger) bei Gruppe I 4 mal, bei Gruppe II 8mal.

4. Gleichzeitige starke Verkürzung der Hördauer für $c_{4}$ und $c_{5}$ (in dem eben erwähnten Grade) bestand bei Gruppe I 1 mal, bei Gruppe II $7 \mathrm{mal}$.

5. Die obere Tongrenze war niemals eingeengt.

6. Die untere Tongrenze war bei Gruppe I einmal eingeengt, bei Gruppe II zweimal, natürlich als Ausdruck der Mittelohraffektion ( 1 mal $\mathrm{C}$, einmal $\mathrm{c}_{1}$ ).

7. Die Knochenleitung ist bei Gruppe I stets verkü rzt, darunter - von einem Fall abgesehen - stets auf $60 \%$ und weniger. Bei Gruppe IT ist sie 10 mal verkürzt (stets auf $60 \%$ und weniger), 3 mal hingegen infolge des Einflusses der gleichzeitigen Mittelohraffektion verlängert. Es ist zu bemerken, daß die Fälle mit verlängerter Knochenleitung trotzdem eine hochgradige Verkürzung der Hördauer für die hohen Töne aufweisen.

8. Die Hörfähigkeit für Flüstersprache ist bei Gruppe I nur I mal gesunken (von $8 \mathrm{~m}$ auf $6 \mathrm{~m}$ zugewandt unsicher), hingegen 6 mal bei Gruppe II (je einmal von 2,5 auf $2 \mathrm{~m}$, von 4 auf $1 \mathrm{~m}$, von 8 auf $6 \mathrm{~m}$ zugewandt, von 8 auf $6 \mathrm{~m}$ abgewandt unsicher, je einmal wurden bei sonst gleichgebliebener Hörfähigkeit für Flüstersprache die hohen oder tiefen Töne schlechter gehört). Unter diesen 7 Fällen war 5 mal die Hördauer hoher Töne stark verkürzt und zwar auf beiden Seiten oder nur auf dem bezüglich des Sprachgehörs geschädigten Ohre; hier ist also in dem inneren Ohre die Ursache der Schallschädigung zu suchen. Nur zweimal (in je einem Fall der Gruppe I und II) war auf dem bezüglich des Sprachgehörs geschädigten Ohre die Hördauer für die hohen Töne besser als auf dem zweiten $\mathrm{Ohr}$; hier war also, zumal auch 
die tiefen Zahlen schlechter als die hohen gehört wurden, die gleichzeitige Mittelohraffektion als Ursache der Schädigung des Sprachgehörs anzusehen.

9. Von den unter Nr.4 erwähnten 7 Fällen der Gruppe II zeigen fünf sichere Kennzeichen einer früher überstandenen Mittelohreiterung (Narben, Perforation), einmal bestand eine starke Einziehung; die Fälle von Verkalkung - es waren nur Leute mit umfangreicher Verkalkung ausgewählt - zeigten also im allgemeinen nur geringere Schädigungen bezüglich der Hörfähigkeit hoher Töne.

10. Was die befallene Seite anbelangt, so ist bei Gruppe I die rechte Seite häufiger bzw. stärker befallen ( 7 mal), wesent. lich seltener die linke ( $3 \mathrm{mal})$, gleichfalls erheblich seltener sind beide Seiten gleich stark betroffen $(3 \mathrm{mal})$. Bei Gruppe II ist hingegen ausnahmslos diejenige Seite stärker befallen, die die eingangs genannten pathologischen Residuen (Narben usw.) auf wies (9 mal). Nur einmal zeigt ausnahmsweise das von pathologischen Veränderungen freie Ohr die stärkere Schädigung; in diesem Falle war aber dieses zweite Ohr in früheren Jahren von einem Kopftrauma betroffen worden. Wesentlich seltener (5 mal) zeigten beide Ohren eine gleich starke Schädigung.

Schlußurteil. Die erzielten Resultate sprechen durch ihre Gleichmäßigkeit eine deutliche Sprache. Sie zeigen unabweisbar, daß beim Menschen das, Bestehen von Mittelohrentzündungen eine Prädisposition schafft für die Entstehung von Schallschädigungen. Man könnte aus den vorliegenden Ergebnissen auch den Schluß ziehen, daß die Ursache dieser Prädisposition das Hinterbleiben geringer Schädigungen der Schallleitungskette ist. Man muß aber hier in seinem Urteil größte Zurückhaltung üben, denn es ist keineswegs auszuzchließen, da $B$ die hinterbliebene Störung des Schalleitungsapparates bedeutungslos ist, die abgelaufene Mittelohrentzündung vielmehr eine Vulnerabilität des peripheren Neurons hinterlassen hat: Denn wenn ohne Komplikationen und ohne besondere Schwere abgelaufene akute Mittelohrentzündungen im allgemeinen nachweisbare Schädigungen der Schnecke auch nicht hinterlassen, so wissen wir doch, daß im Höhestadium einer akuten Mittelohrentzündung eine Beteiligung der hohen Töne ziemlich regelmäBig feststellbar ist. Es ist also nicht auszuschließen, daß es sich bei Gruppe II um Schallschädigungen bei bereits vorher vorhanden gewesener Vulnerabilität des peripheren Neurons handelt. Träfe diese letztere Auffassung zu, dann würden die vorstehenden Ergebnisse nicht gegen die Annahme sprechen, daß gänz- 
liche Unterbrechung der Schalleitungskette einen Schutz gegen Schallschädigungen gewährt. Man wird also bei dem geringen Grad der fraglichen Schalleitungsstörungen verallgemeinernde Schlüsse - Bedeutung der Luft- und Knochenleitung, Einfluß schwererer Schädigung der Kette durch Unterbrechung oder Ankylose - zu unterlassen haben. Jedenfalls ist aber für den Begutachter die Feststellung, daß entzündliche Erkrankungen des Mittelohres die Entstehung von Schallschädigungen fördern, von Wichtigkeit, da derartige Beurteilungen häufig vor das Forum des begutachtenden Ohrénarztes kommen. Auch für die Frage der Prophylaxe ergeben sich aus vorstehenden Erörterungen gewisse Hinweise. 